\title{
Preparación para la vida adulta independiente: un camino para promover la inclusión socioeducativa de escolares con necesidades educativas especiales
}

\section{Preparation for independent adult life: a way to promote the socio-educational inclusion of schoolchildren with special educational}

\author{
Karel Llópiz Guerra* (D) \\ Universidad Central "Marta Abreu" de Las Villas, Cuba. \\ ORCID: http://orcid/0000-0002-1500-8000 \\ Diana Estela Pérez Chávez \\ Universidad Central "Marta Abreu" de Las Villas, Cuba \\ ORCID: http://orcid.org/0000-0001-5245-2496 \\ Beatriz Rodríguez Rodríguez \\ Universidad Central "Marta Abreu" de Las Villas, Cuba. \\ ORCID: https://orcid.org/0000-0002-1318-5425
}

Received 06-12-20 Revised 07-25-20 Accepted 09-13-20 On line 09-30-20

*Correspondence

Email: kllopiz@uclv.cu
Cite as:

Llopiz, K., Pérez, D., \& Rodríguez, B. (2020). Preparación para la vida adulta independiente: un camino para promover la inclusión socioeducativa de escolares con necesidades educativas especiales. Propósitos y Representaciones, 8 (SPE3), e733. Doi: http://dx.doi.org/10.20511/pyr2020.v8nSPE3.733 


\section{Resumen}

La inclusión socioeducativa de las personas con discapacidad como proceso desde diferentes contextos, a partir del aprendizaje y la propuesta de acciones educativas que se centren en el desarrollo de habilidades, es un reto de los sistemas educativos en la actualidad, se necesita la eliminación de barreras para la participación, favoreciendo una vida adulta independiente con calidad. Desde la escuela y con el apoyo de las familias se deben proporcionar los medios adecuados para que los escolares con necesidades educativas especiales adquieran las capacidades sociales y la autonomía personal para interactuar de manera adecuada, para aumentar las probabilidades de éxito de su inclusión. En el presente artículo se realiza una sistematización acerca de la preparación para la vida adulta independiente de escolares con necesidades educativas especiales, como premisa para proponer las acciones didácticas metodológicas que favorezcan su inclusión socioeducativa.

Palabras claves: Inclusión socioeducativa; Discapacidad; Acciones didáctico-metodológicas; Vida adulta e independiente.

\section{Summary}

The socio-educational inclusion of people with disabilities as a process from different contexts, based on learning and the proposal of educational actions that focus on the development of skills, is a challenge of education systems today, the removal of barriers is needed for participation, favoring an independent adult life with quality. From school and with the support of families, adequate means must be provided for schoolchildren with special educational needs to acquire social skills and personal autonomy to interact appropriately, to increase the likelihood of their inclusion success. This article aims to offer methodological teaching actions to teachers and specialists to promote the development of independent adult life in schoolchildren with special educational needs who are in a process of socio-educational inclusion.

Keywords: Social and Educative Inclusion; Disability; Didactic and Methodological Actions; Independent Adult Life

\section{Introducción}

La preparación para la vida adulta independiente de las personas con discapacidad constituye un proceso en el que influye el desarrollo de actividades y acciones por parte de los escolares, pero sobre todo la identificación y propuesta de oportunidades y la determinación de los recursos y apoyos que harán realidad el proceso hacia la mayor autonomía personal posible, sin excluir la cooperación escolar, familiar y social. Se requiere mantener expectativas positivas, confianza en sus posibilidades y respeto a su autodeterminación y a sus derechos.

La Convención Internacional sobre los Derechos de las Personas con Discapacidad (Organización de Naciones Unidas, 2006) establece que las personas con discapacidad son titulares de derechos y que los poderes públicos están obligados a garantizar el ejercicio pleno y efectivo de estos derechos. Orientada por los principios de participación e inclusión, no discriminación e igualdad de oportunidades, la Convención establece claramente los derechos a la inclusión educativa y laboral, a la vida independiente y a la participación en la comunidad, entre otros (Fullana, Pallisera, Vilà, Montserrat \& Rovira, 2016).

Para alcanzar una total inclusión socioeducativa es necesario, desde diferentes contextos, la eliminación de barreras para la participación y el aprendizaje y la propuesta de acciones educativas que se centren en el desarrollo de habilidades para una vida adulta independiente. 
Encontrar puntos de vista comunes en diferentes ámbitos educativos permite unificar y proponer acciones y estrategias que favorezcan este importante proceso, del que saldrán beneficiados tanto los escolares, como la familia y la sociedad.

Se hace necesario que en la escuela y desde las familias se proporcionen los medios adecuados para que los escolares con necesidades educativas especiales adquieran las capacidades sociales y de autonomía personal necesarias para interactuar de manera adecuada, para aumentar las probabilidades de éxito de su inclusión, para aceptar y ser aceptado por los que lo rodean.

Se deben impulsar programas educativos más funcionales, en respuesta a las necesidades individuales, con contenidos fundamentales para el desarrollo de una vida autónoma (un currículo para la vida). En este sentido, tanto las personas con discapacidad como los profesionales valoran especialmente que la formación pueda ayudarles a ganar confianza y autonomía. (Pallisera, M. et al: 2018)

En las "Metas inclusivas" para 2021 se define que la escuela inclusiva apuesta por la participación, el respeto mutuo, el apoyo, la sensibilidad y el reconocimiento de todos los individuos (Marchesi, Blanco, Hernández, 2014).

Se sostiene que la educación inclusiva es la base para la visibilización, aceptación y valoración de la diversidad como elemento esencial para la transformación de los sistemas sociales. Garantizar el derecho a la vida independiente requiere previamente haber garantizado el acceso a la educación con calidad, que es la que permite que todas las personas puedan alcanzar al máximo nivel posible el libre desarrollo de su personalidad. (Tosi, Peláez, D’Orazio, Larrañaga, Saavedra \& Baeza, 2016).

En el presente artículo se realiza una sistematización acerca de la preparación para la vida adulta independiente de escolares con necesidades educativas especiales, como premisa para proponer las acciones didácticas metodológicas que favorezcan su inclusión socioeducativa.

\section{Papel de la educación}

A pesar de que casi todas las personas aceptan la importancia del trabajo para el desarrollo de una vida adulta independiente, pocos reconocen que esta aspiración guarda relación y emana del fin y los objetivos de la educación.

Tanto el fin como los objetivos generales de la educación poseen largo alcance y sus aspiraciones confirman la intención de alcanzar logros que se materializarán en la etapa de la adultez del ciudadano, con total independencia y autonomía.

Este fin y objetivos generales y sus particulares derivaciones alcanzan a los escolares con necesidades educativas especiales. Para ellos, estas aspiraciones se magnifican pues se trata de lograr la mayor calidad de vida posible, a partir de las demandas sociales, en un contexto determinado.

El fin y los objetivos generales de la educación especial hallan un contexto muy favorecedor a través del sistema social y la política educacional con la preocupación manifiesta, por todos y cada uno de sus ciudadanos, sin excluir a aquellos con necesidades educativas especiales asociadas o no a discapacidad (Borges, 2014) 
En Cuba, en respuesta a los fines y objetivos generales de la educación y a los de cada nivel de enseñanza en particular la Preparación para la vida adulta independiente y socio - laboral posee una singular importancia.

\section{La formación del profesorado}

Ante el reto del perfeccionamiento de la formación de pregrado y posgrado de los profesionales de la Educación Especial, se presentan las bases teórico-metodológicas para la atención a la diversidad y la inclusión educativa, a partir de la precisión de conceptos y procedimientos, orientados a la reflexión en el ámbito de la Educación Especial como concepto generalizador y ofrecer las herramientas cognitivas, procedimentales y actitudinales, para la contextualización de la Didáctica en la Educación Especial como aspecto esencial de la concepción potenciadora del desarrollo de estos escolares. (Girado \& Martin, 2017)

Las universidades, como entes formadores de docentes en educación especial, son los mayores responsables en su idónea capacitación, no solo en el buen dominio temático de los planes educativos y de atención para adultos con discapacidad, sino también en la obligatoriedad y necesidad que retoma incluir a la familia en cualquier trabajo con la persona en esta condición. (Madrigal-Lizano 2015)

Desde la perspectiva de la pedagogía de la diversidad no sólo se reconoce las diferencias de los educandos, sino también de los profesores, sobre todo las relacionadas con su preparación que condiciona la variedad de métodos, medios y procedimientos que los mismos emplean en función de una educación para todos. (Espino, Insua, 2018)

En este empeño resulta fundamental preparar a los docentes mediante acciones didácticas y metodológicas que preparen a las personas en condiciones de inclusión y así puedan llevar una vida más autónoma e independiente, ello debe plantearse a lo largo de toda la vida, el trabajo por el desarrollo de una vida adulta independiente es de vital importancia desde las primeras edades.

Sobre la existencia de una Didáctica Especial o para la Educación Especial, no existe un consenso teórico, se asume el carácter singular de la Didáctica para la atención a los escolares con necesidades educativas especiales asociadas o no a discapacidades, dentro de la particularización del proceso de enseñanza-aprendizaje (objeto de estudio de la Didáctica General) para las diferentes esferas del conocimiento (Didácticas Particulares).

Desde la sistematización del uso de los recursos didácticos, mediadores para el desarrollo y enriquecimiento del proceso de enseñanza-aprendizaje para la movilización cognitiva, afectiva y vivencial de los escolares con necesidades educativas especiales, la orientación, la administración de las ayudas pedagógicas, y por tanto, el ajuste de la respuesta educativa; ello da carácter singular al conjunto de actuaciones transformadoras y formativas (decisiones y modelación del proceso de enseñanza-aprendizaje) que se asumen en condiciones de desarrollo más complejas, en un clima comunicativo e interactivo (Girado \& Martin, 2017).

El proceso de inclusión se encamina a la reflexión y la necesidad de su puesta en práctica, poder examinarla e intentar desarrollar una cultura más inclusiva.

La misma no puede desvincularse del contexto en el que se desarrolla, ni de las relaciones sociales que se establecen y pueden sostener o no ese desarrollo. Es en la compleja interacción entre los individuos que evolucionan las convicciones, los valores comunes y donde se producen los cambios.

Es imposible separar estas convicciones de las relaciones en que se expresan, si bien es importante empezar desde la y así lo exige la sociedad, preparar a un hombre para su plena 
incorporación a la vida social, resultando imprescindible su transformación como ser humano, distinguiéndose su papel activo, a partir del desarrollo de las habilidades y capacidades que haya podido alcanzar hasta ese momento (Llerena, Salinas, 2018).

Cuando se hace alusión a vida adulta independiente se refiere a que la persona sea dueña de su propia vida, que decida sobre las cosas que le preocupan y le interesan.

Las concepciones actuales son el resultado de la transformación de diferentes modelos que durante su proceso de desarrollo han alcanzado posiciones cada vez más progresistas e inclusivas.

Por lo que se recomienda en el orden metodológico y didáctico que se tenga en cuenta el perfil de necesidades de apoyo que viene determinado por la identificación de los apoyos necesarios para mejorar el funcionamiento individual.

Para ello el docente debe identificar el tipo de apoyo necesario, la intensidad de apoyo y la persona responsable de proporcionar el apoyo en cada una de las nueve áreas tales como:

1) desarrollo humano

2) enseñanza y educación

3) vida en el hogar

4) vida en la comunidad

5) empleo

6) salud y seguridad

7) conductual social

8) protección y defensa.

\section{Proceso de transición a la vida adulta. Aspectos claves}

La transición a la vida adulta constituye una etapa fundamental en la vida de las personas. En ella se van a sentar las bases del proyecto de vida. Coincide con el final del proceso educativo en las instituciones escolares e influye en la futura organización de la vida adulta.

El resultado de la transición desde la adolescencia es la emergencia de un adulto que va tomando responsabilidades crecientes sobre sus actos y los resultados de los mismos, que se mueve hacia una independencia cada vez mayor de sus padres y su familia en lo que se refiere a la toma de decisiones, y que está destinado a tomar su lugar en la sociedad como miembro activo de varios subgrupos dentro de su ambiente. A pesar de que la transición de jóvenes con discapacidad a la vida adulta y al mundo del trabajo es objeto de gran interés desde hace décadas, la generalización de programas y prácticas positivas para favorecer ese proceso es muy escasa e inexistente en muchos lugares. Por ello, es relevante promover e incentivar prácticas innovadoras que sean de fácil generalización, y que materialicen modelos centrados en la persona y en su calidad de vida.

Díaz, M. et al. (2013) señalan que en este proceso se ofrecen oportunidades de formación de diferente tipo, y se van descubriendo las alternativas laborales y de desarrollo de la carrera y proyecto personal. Esta etapa sienta las bases de cómo la persona va a desenvolverse en diferentes ámbitos vitales como el empleo, ocio, formación continua, cuidado personal, interacción social y participación en la comunidad. 
El proceso de transición a la vida adulta debe responder a una planificación explícita, pero en el caso de las personas con discapacidad, y tanto más cuanto más severas sean estas, es necesario una planificación detallada, pues solo a través de una programación coordinada y continuada es posible que los estudiantes con limitaciones importantes puedan integrarse en el mercado laboral y en la sociedad.

Se debe recomendar al personal docente que deben tener en cuenta los principales problemas en la transición de jóvenes con discapacidad en condiciones de inclusión a partir de:

- Reducir o prevenir las altas cifras de alumnos que abandonan la educación y de desempleados jóvenes.

- Acceder a la educación de calidad y aumentar la formación de los jóvenes.

- Ofrecer cualificaciones adecuadas que correspondan con las capacidades de los jóvenes y les permita enfrentarse adecuadamente a la vida adulta y laboral.

- Fomentar el contacto y el entendimiento mutuo entre los ambientes educativos y laborales.

Se destacan como aspectos claves que es necesario considerar en la transición los siguientes:

a. Entenderla como un proceso que debe ser apoyado por la existencia e implementación de medidas legislativas y políticas.

b. Debe asegurar la participación del joven y respetar sus opciones personales donde el joven, su familia y los profesionales deben trabajar juntos para elaborar un programa individual.

c. La elaboración de un programa educativo individual (adaptación curricular individual) centrado en el progreso del joven y en cualquier cambio realizado en su situación escolar debe ser parte obligatoria del proceso de transición.

d. Ha de basarse en la participación y colaboración directas de todas las partes involucradas.

e. Requiere una estrecha cooperación entre los centros escolares y el mercado laboral para que los jóvenes experimenten condiciones laborales reales.

f. Finalmente, la transición es parte de un largo y complejo proceso de preparación y facilitación para que los jóvenes se inicien en la vida económica adulta.

El proceso de transición hacia una vida adulta independiente debe culminar en la obtención no solo de un empleo útil y una actividad digna y remunerada, sino también en el máximo nivel de autonomía personal y autodeterminación, y acceso al rango de adulto en sus diversos roles. Debe, por ello, conducir al desarrollo y mantenimiento de relaciones interpersonales y sociales, así como a la participación en la vida comunitaria y en actividades de tiempo libre, sin olvidar el bienestar emocional de la persona y la definición de su convivencia y el rol familiar futuro.

Jordán de Urriés y Verdugo (2013), siguiendo a Wehman (2002), determina que son tres los elementos esenciales que han de configurar cualquier proceso de transición desde la escuela a la edad adulta:

a) un programa educativo con aprendizajes significativos y útiles 

adulta;

b) un plan individualizado que contenga los componentes principales que llevan a la vida

c) opciones de empleo, vivienda y participación en la comunidad que posibiliten a los estudiantes y sus familias la mejor elección.

Wehman (2002) concreta estos componentes en los siguientes términos (recogidos por Jordan de Huríes y Verdugo, 2013):

Los programas educativos deben ofrecer la oportunidad de aprender junto a jóvenes sin discapacidad y su planificación comienza en los niveles más elementales, implicando a todos los profesores a lo largo del proceso educativo.

El currículo profesional debe secuenciarse a lo largo de los diferentes niveles para ir asegurando el adecuado avance de un nivel a otro. Este currículo debe ser funcional, en correspondencia con las demandas específicas del escolar con respecto al empleo, reflejando habilidades laborales demandadas en el empleo y ha de incluir situaciones variadas, basadas en las ofertas de empleo en la comunidad disponibles para todos.

Los entrenamientos educativos deben realizarse en lugares de trabajo reales e incluir habilidades laborales, ratios de producción y habilidades interpersonales. Los profesores de apoyo, especialistas en educación especial, y especialistas en empleo han de trabajar juntos con este objetivo, siendo necesario la puesta en funcionamiento de servicios específicos de preparación profesional que se desarrollen con tiempo suficiente antes de terminar el proceso escolar. Un aspecto relevante consiste en centrarse en los posibles trabajos a desarrollar y en definir las estrategias de entrenamiento, desarrollando todo ello en el máximo nivel de inclusión posible con iguales sin discapacidad.

En este proceso debemos implicar en lo posible a los padres y hemos de identificar a los profesionales y servicios que puedan proporcionar formación continuada en el empleo. Para esto, es necesario disponer de un coordinador que integre el programa, los servicios disponibles, los profesionales implicados, y los recursos de la comunidad, y también que se desarrolle y realice la evaluación de resultados.

Los investigadores consideran la vida adulta independiente y socio-laboral como un proceso en el que el escolar con necesidades educativas especiales alcanza en la adultez, un mayor nivel de independencia en los diferentes contextos en los que interactúa, a partir de programas orientados a facilitar el desarrollo de la independencia, la autonomía personal y la integración laboral (Vega, Gómez, Fernández, 2013). Otro aspecto se refiere a que la posibilidad de acceso creciente a la universidad, condiciona que este espacio debe establecer los ajustes a sus dinámicas que permitan la permanencia de todos los alumnos. De esta manera, la educación superior se convierte en un componente del sistema de influencias que propicia el desarrollo de las potencialidades de los estudiantes con necesidades educativas especiales y su posterior inserción en el mercado laboral (Pérez, 2017).

\section{Recomendaciones didáctico-metodológicas desde la experiencia práctica}

El equipo de investigadores propone las siguientes recomendaciones didáctico-metodológicas generales para la transición hacia una vida adulta e independiente donde debe tenerse en cuenta:

- Debe partir de las demandas del contexto.

- Comenzar con el diagnóstico de las necesidades de cada escolar. 
- Se tomarán en consideración las capacidades del escolar con necesidades educativas especiales y no sus limitaciones.

- La preparación para la vida adulta independiente y socio-laboral de los escolares con necesidades educativas especiales es posible, pero demanda la coordinación entre la escuela, familia y comunidad.

- Desde los programas de las asignaturas, los círculos de interés, el trabajo pioneril y otros espacios se deben priorizar, para los estudiantes con necesidades educativas especiales, los contenidos funcionales que contribuyan al desarrollo de la vida adulta independiente y socio-laboral.

- El cumplimiento de las acciones se debe realizar en un entorno escolar, familiar y comunitario normalizador.

- Priorizar, en el trabajo con escolares con necesidades educativas especiales, el desarrollo de habilidades polivalentes.

Los componentes para el trabajo con esta área, están sujeta a determinado grado de variabilidad y su selección, organización y jerarquización guarda una estrecha relación con el contexto (comunitario, escolar y familiar) en que se desarrolle el escolar con necesidades educativas especiales, las demandas de dicho contexto y las características individuales del escolar.

No obstante, a la variabilidad de los componentes de esta área, se centra la atención en los siguientes: el cuidado y desarrollo personal, el hogar y la comunidad, la preparación sociolaboral y el tiempo libre.

A continuación, se sugieren las acciones didáctico-metodológicas para cada uno de estos componentes:

Acción 1: Favorecer situaciones de aprendizaje para el cuidado y desarrollo personal que tengan como referente la vida cotidiana y se desarrollen en marcos reales.

Con el cuidado y desarrollo personal la higiene es un aspecto imprescindible a trabajar con los niños en condiciones de inclusión, a partir de hacerles comprender y desarrollar como hábitos para su vida cotidiana el lavado de las manos, cepillado de los dientes, lavado de la cara, del cabello, sus órganos genitales y su cuerpo en general.

Operaciones: creación de condiciones propicias para el logro de hábitos relacionados con la higiene personal (el lavado de las manos, cepillado de los dientes, lavado de la cara, lavado del cabello, lavado de los órganos genitales, lavado del cuerpo).

- Desarrollo de rutinas para vestirse y desvestirse (Selección de la ropa, abotonar y desabotonar, atar el calzado).

- Creación de condiciones para el descanso (Cuándo descansar; dónde descansar, cómo descansar)

- Planteamiento de problemas y situaciones de la vida diaria relacionadas con la organización de la economía personal (Uso del dinero: papel y moneda; distribución del dinero según necesidades, pago de cuentas, compras, ahorro)

- Realización de actividades prácticas fuera de la institución educacional que favorezcan la orientación en la comunidad, fuera de la comunidad, comprender direcciones, solicitar direcciones, ofrecer direcciones, el uso del transporte, conocimiento de las normas de circulación.

Acción: 2.- Crear espacios simulados en la institución educativa y sus alrededores que propicien el desarrollo de habilidades para el desenvolvimiento en el hogar y la comunidad. 


\section{Operaciones}

- Realización de actividades prácticas para el aprendizaje de las labores en el hogar, como: la elaboración de alimentos, fregado de utensilios, organización y limpieza del hogar, lavado de ropas, planchado de ropas, reciclaje de la basura, selección y utilización adecuada de productos químicos y peligrosos.

- Realización de actividades demostrativas dirigidas a la satisfacción de necesidades personales, como: demostración de hábitos alimentarios, uso de equipos electrodomésticos, uso del teléfono, uso adecuado de los medios audiovisuales.

- Aprovechamiento de las potencialidades que brindan tanto las actividades curriculares como extracurriculares para contribuir a la preparación para la vida en pareja: relaciones afectivas, comunicación con la pareja, conducta sexual responsable, higiene de la vida sexual, salud sexual y reproductiva, formación de una familia, tenencia y cuidado de los hijos.

- Empleo de la transversalidad en el currículo para el trabajo con los ejes temáticos de educación para la salud: uso de medicamentos, prevención de drogas, autoestima, normas de convivencia.

- Creación de condiciones para la participación en actividades comunitarias

- Asistencia a reuniones y actividades de las diferentes organizaciones de la comunidad, el barrio.

- Asistencia a labores de saneamiento y embellecimiento del ornato.

- Participación en exposiciones y festivales comunitarios.

Acción 3.-Identificar las diferentes potencialidades que brinda el entorno para favorecer la preparación socio-laboral.

\section{Operaciones:}

- Exploración de posibilidades laborales: Visitas a centros laborales de la comunidad, demostrar habilidades específicas para un empleo, ambiente laboral.

- Selección de oportunidades laborales: demostración de modos de actuación laborales, dominio de normas jurídicas para el empleo, seguir las normas de higiene, seguridad y protección del trabajo, mantenimiento de un empleo, conocimiento de aspectos esenciales acerca del peligro, las vulnerabilidades y los riesgos laborales.

- concepción de estrategias que favorezcan el desarrollo de habilidades comunicativas y conversacionales para la correcta interacción social.

Acción 4-Identificación de diferentes ofertas del entorno para la participación en actividades de ocio y tiempo libre.

- Elección de actividades de ocio y apropiación de modos de actuación de acuerdo a la actividad de ocio elegida.

- Utilización de las posibilidades del ambiente en que se encuentra para la utilización del tiempo libre.

Para desarrollar estas acciones con éxito es necesario la movilización de apoyos dentro y fuera de la escuela. 
La primera tarea para establecer apoyos efectivos consiste en movilizar aquellos recursos que ya existen, tanto dentro como fuera de las escuelas. En muchos casos esto será lo único que hay que hacer para atender las necesidades de una amplia gama de estudiantes. No es posible decidir qué apoyos adicionales son necesarios a menos que se empleen al máximo los recursos ya disponibles en las escuelas.

Se organizan en el distrito o comunidad estrategias de trabajo y apoyo escuelas entre escuelas, se intercambian experiencias y formas de trabajo, se crean servicios itinerantes con los que cada escuela va contando.

El modelo de servicios itinerantes tiene la ventaja de generar servicios especializados accesibles directamente a todas las escuelas y comunidades.

Cualquiera sea la forma en que se organicen los apoyos, es importante que involucren la colaboración multisectorial - esto es, el trabajo conjunto entre una serie de servicios e instancias.

Esto significa que el especialista que trabaja directamente con los estudiantes, las familias y las escuelas debe ser capaz de imbuir su trabajo con una perspectiva interdisciplinaria.

Debe organizarse desde la puesta en práctica de un currículo que tenga presente a todos los alumnos, esto implica una serie de estrategias de enseñanza a partir de:

- Lograr la participación del alumno, motivarlo, utilizar sus intereses.

- Utilizar técnicas de trabajo individual y grupal.

- Crear y adaptar materiales y recursos variados.

- Organizar el proceso docente y extradocente.

- Vincular la enseñanza-aprendizaje con la vida para abordar la solución de problemas reales.

- Planificar las clases acorde a las características de los alumnos (dosificar contenidos, actividades, materiales y la evaluación de los objetivos del currículo

En la organización no pueden faltar las exigencias flexibles, la creatividad, y la cooperación, además de considerar la creación de espacios compartidos; el establecimiento de agrupamientos flexibles; horarios flexibles; espacios de atención individualizada; el desarrollo de la responsabilidad en los alumnos; el establecimiento de normas y formas de trabajo, los servicios de apoyo al centro y la accesibilidad de espacios físicos y a materiales.

\section{Conclusión}

La preparación para la vida adulta independiente de los escolares con necesidades educativas especiales constituye una condición necesaria para el logro de su inclusión socioeducativa, en ella es imprescindible contar con recursos y apoyos del entorno, con la familia y trabajar en función de sus necesidades y capacidades.

Las acciones didácticas metodológicas que se ofrecen en este artículo contribuyen a la preparación de los maestros y especialistas para saber enfrentar con iniciativas la solución de los problemas de la práctica pedagógica y contribuir al logro de la vida adulta independiente en los niños, adolescentes y jóvenes con necesidades educativas especiales.

\section{Referencias}

Borges, S. (2014). Inclusión educativa y Educación Especial: un horizonte singular y diverso para 
igualar las oportunidades de desarrollo. La Habana: Sello Educación cubana.

Espino-Morales L, A. I. (2018). La superación del profesional y la inclusión educativa: binomio necesario para la atención a la diversidad. Revista Varela, 15-21.

Fullana, N., J, P., Montserrat, S., \& Rovira, P. (2016). las personas con discapacidad intelectual como investigadoras. Debate, retos y posibilidades de la investigación inclusiva. EMIPIRIA. Revista de Metodología de Ciencias Sociales, 111-138.

Guirado, V., \& Martin, D. (2017). Bases teórico-metodológicas para la atención a la diversidad y la inclusión educativa. Revista Universidad y Sociedad, 45-52.

Díaz, M. et al. (2013). Formación para la autonomía y la vida independiente. Guía general. https://www.sindromedown.net/wp-content/uploads/2014/09/144L_guia.PDF

LLerena, O., \& Salinas, C. (2018). Inclusión educativa y adaptaciones curriculares en el proceso de enseñanza aprendizaje. Ecuador: Redes 2017.

Madrigal-Lizano, A. (2015). El papel de la familia de la persona adulta con discapacidad en los procesos de inclusión laboral. Revista electrónica Educare, 197-211.

Marchesi, B. H. (2014). Avances y desafíos de la educación inclusiva en Latinoamérica.

Metas educativas 2021. Organización de Estados Iberomericanos.

Pérez, L. (4 de marzo de 2017). Obtenido de http://revgmespirtuana.sld.cu/article/view/513

Tosi, I., Pelaez, L., \& D Orazio, L. (2016). Educación inclusiva y vida independiente. Revista Universitaria, 134-164.

Vega, C., Gómez, M., \& Fernández, R. (2013). El papel del contexto educativo en la autodeterminación. Análisis de su influencia en el proceso de transición a la vida adulta. Revista iberoamericana de Educación, 19-25. 